
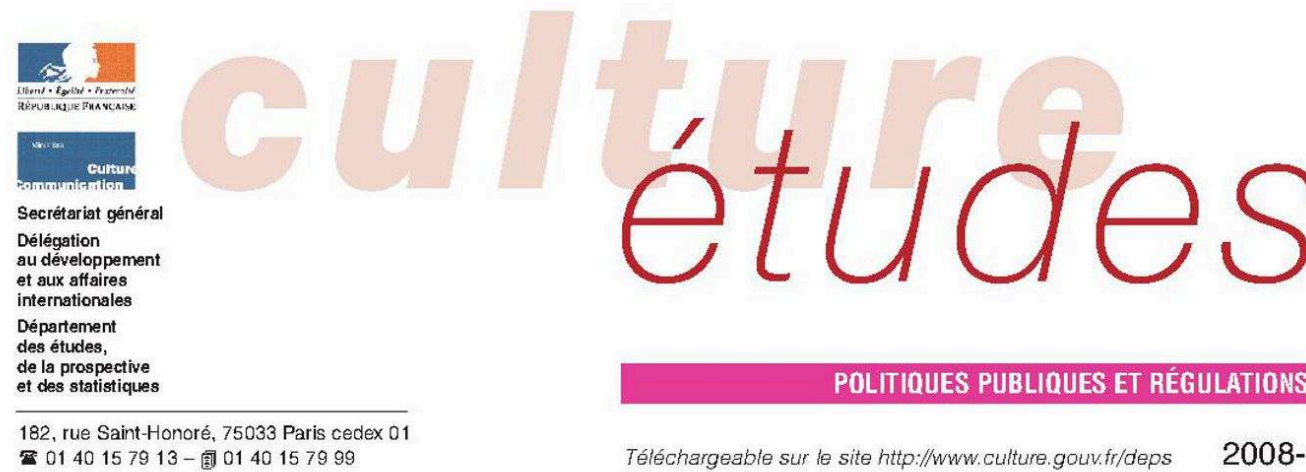

\title{
La gratuité des musées et des monuments : qu'en pensent les publics en France* ?
}

Anne Gombault, Caroline Urbain, Dominique Bourgeon-Renault, Marine Le Gall-Ely, Christine Petr**

\section{Avant-propos}

Les formes de gratuité sont au cour des réflexions des politiques culturelles. La gratuité fait l'objet d'expérimentations, utilisant diverses formes (cartes, chèques, abonnements forfataires, ciblage...) dont certaines ont fait l'objet d'évaluation. Ces gratuités sont en revanche moins étudiées sous l'angle de leur réception par les publics que ce sout sur le registre de la politique - la «politique de gratuité »-ou sur celui d'une mesure particulière. C'est le mérite de cette synthèse que d'éclairer la question de la gratuité par celle de sa perception par les publics. Cette perception manifeste une grande complexité, inhérente à la pluralité des dimensions à prendre en compte pour atteindre des objectifs culturels, marketing, économiques. Elle traduit que le prix de la culture, même égal à zéro, est d'abord une question de valeurs.
La gratuité dans les musées et les monuments est un sujet de débat à la fois ancien - elle fut considérée comme une valeur fondamentale au moment où ils furent créés - et récurrent, puisqu'elle est encore centrale dans leurs plus récentes mutations ${ }^{1}$.

Parmi toutes les questions posées à son sujet, celle de ses représentations par les publics semble absente. Et pourtant, en 2000 , lorsque fut évaluée la mesure du premier dimanche de chaque mois gratuit au Louvre après deux années de fonctionnement, la question des représentations avait été formulée en ces termes : « Cet idéal de partage de la culture est-il porté également par le public $^{2} ? »$

Alors que la question de l'impact de la gratuité a été abondamment documentée en termes de fréquentation ${ }^{3}$,

Philippe Chantepie

* Ce texte est extrait des résultats d'une recherche principalement qualitative financée par le Deps et récemment publiée : voir Anne Gom bault, Christine Petr, Dominique Bourgeon-Renault, Marine Le Gall, Caroline Urbain, la Gratuité des musées et des monuments, côté publics, Paris, DePs, Ministère de la Culture et de la Communication/La Documentation française, 2006.

** Anne Gombault est professeur à Bordeaux Management School (Bem) où elle dirige la chaire Arts, cul ture et management en Europe qui réunit une dizaine de chercheurs en management des arts et de la culture. Caroline URBAIN est maître de conférences à l'université de Nantes, chercheur au CRGNA. Dominique BOURGEON-RENAULT est professeur à l'université de Bourgogne, chercheur au CERMAB-LEG, UMR CNRS 5118 et chercheur associé à la chaire Arts, culture et management en Europe (BEM). Marine LE GALL-ELY est maître de conferences à l'université de Rennes 2 et chercheur au sein du CREss-Lessor EA2614, Rennes 2 et de l'ICl, EA 2652, Ubo. Christine PETr est maître de conférences à l'universite de Rennes 1, et chercheur au CREM, UMr CNRS 6211.

1. Loi $n^{\circ} 2002-5$ du 4 janvier 2002 relative aux musées de France (parue au $J O, n^{\circ} 4,5$ janvier 2002).

2. Claude FOURTEAU, «La politique des publics au Louvre 》, Publics et projets culturets, Paris, L'Harmattan coll "Patrimoines et socítés", $n^{\circ} 46,2000$.

3. Voir Sylvie OCJOBRE et François RoveT, «La gratuité du premier dimanche du mois dans les monuments et muśés nationaux », dans François RouEt (sous la dir. de), les Tarifs de la culture, Paris, DEPS, Ministère de la Culture et de la communication/La Documentation française, 2002, chap. VI, p. 303-337. 


\section{La gratuité des musées et des monuments : qu'en pensent les publics en France?}

Anne Gombault, Caroline Urbain, Dominique Bourgeon-Renault, Marine Le Gall-Ely et Christine Petr

Éditeur : Département des études, de la prospective et des statistiques

Lieu d'édition : Paris

Année d'édition : 2008

Date de mise en ligne : 8 juillet 2015

Collection : Culture études

ISBN électronique : 9782111398818

\section{Qbooks}

http://books.openedition.org

\section{Édition imprimée}

Date de publication : 1 janvier 2008

Nombre de pages : 12

\section{Référence électronique}

GOMBAULT, Anne ; et al. La gratuité des musées et des monuments : qu'en pensent les publics en France? Nouvelle édition [en ligne]. Paris : Département des études, de la prospective et des statistiques, 2008 (généré le 25 avril 2021). Disponible sur Internet : <http://books.openedition.org/deps/276>. ISBN : 9782111398818. 

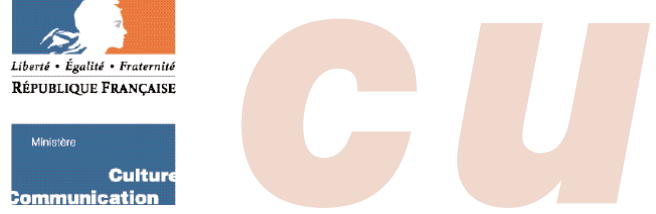

Secrétariat général

Délégation

au développement

et aux affaires

internationales

Département

des études,

de la prospective

et des statistiques

\title{
La gratuité des musées et des monuments : qu'en pensent les publics en France*?
}

\author{
Anne Gombault, Caroline Urbain, \\ Dominique Bourgeon-Renault, Marine Le Gall-Ely, Christine Petr ${ }^{* *}$
}

\section{Avant-propos}

Les formes de gratuité sont au cour des réflexions des politiques culturelles. La gratuité fait l'objet d'expérimentations, utilisant diverses formes (cartes, chèques, abonnements forfaitaires, ciblage...) dont certaines ont fait l'objet d'évaluation. Ces gratuités sont en revanche moins étudiées sous l'angle de leur réception par les publics que ce soit sur le registre de la politique - la «politique de gratuité»- ou sur celui d'une mesure particulière. C'est le mérite de cette synthèse que d'éclairer la question de la gratuité par celle de sa perception par les publics. Cette perception manifeste une grande complexité, inhérente à la pluralité des dimensions à prendre en compte pour atteindre des objectifs culturels, marketing, économiques. Elle traduit que le prix de la culture, même égal à zéro, est d'abord une question de valeurs.
La gratuité dans les musées et les monuments est un sujet de débat à la fois ancien - elle fut considérée comme une valeur fondamentale au moment où ils furent créés - et récurrent, puisqu'elle est encore centrale dans leurs plus récentes mutations ${ }^{1}$.

Parmi toutes les questions posées à son sujet, celle de ses représentations par les publics semble absente. Et pourtant, en 2000, lorsque fut évaluée la mesure du premier dimanche de chaque mois gratuit au Louvre après deux années de fonctionnement, la question des représentations avait été formulée en ces termes : «Cet idéal de partage de la culture est-il porté également par le public $^{2} ? »$

Alors que la question de l'impact de la gratuité a été abondamment documentée en termes de fréquentation ${ }^{3}$,

Philippe Chantepie

\footnotetext{
* Ce texte est extrait des résultats d'une recherche principalement qualitative financée par le DePs et récemment publiée : voir Anne GoMBault, Christine Petr, Dominique Bourgeon-Renault, Marine Le Gall, Caroline Urbain, la Gratuité des musées et des monuments, côté publics, Paris, DEPS, Ministère de la Culture et de la Communication/La Documentation française, 2006.

** Anne GomBAULt est professeur à Bordeaux Management School (BEM) où elle dirige la chaire Arts, culture et management en Europe qui réunit une dizaine de chercheurs en management des arts et de la culture. Caroline URBAIN est maître de conférences à l'université de Nantes, chercheur au CRgna. Dominique BourgeOn-RENAUlt est professeur à l'université de Bourgogne, chercheur au CERMAB-LEG, UMR CNRS 5118 et chercheur associé à la chaire Arts, culture et management en Europe (BEM). Marine LE GALL-ELY est maître de conférences à l'université de Rennes 2 et chercheur au sein du Cress-Lessor EA2614, Rennes 2 et de 1'ICI, EA 2652, UBO. Christine PeTR est maître de conférences à l'université de Rennes 1, et chercheur au CREM, UMR CNRS 6211.

1. Loi ${ }^{\circ} 2002-5$ du 4 janvier 2002 relative aux musées de France (parue au $J O, \mathrm{n}^{\circ} 4,5$ janvier 2002).

2. Claude Fourteau, «La politique des publics au Louvre», Publics et projets culturels, Paris, L'Harmattan, coll. «Patrimoines et sociétés », $\mathrm{n}^{\mathrm{o}} 46,2000$.

3. Voir Sylvie Octobre et François Rouet, «La gratuité du premier dimanche du mois dans les monuments et musées nationaux », dans François Rouet (sous la dir. de), les Tarifs de la culture, Paris, DEPS, Ministère de la Culture et de la communication/La Documentation française, 2002, chap. VI, p. 303-337.
} 
celle de ses représentations ${ }^{4}$ par les publics a fait l'objet de peu de travaux ${ }^{5}$. L'aborder par une recherche principalement qualitative permet donc de comprendre les représentations et les projets d'usage qu'ont en France les publics de la gratuité dans les musées et les monuments et les comportements qui sont les leurs, qu'ils soient visiteurs habitués, occasionnels ou bien encore non-visiteurs.

Que disent les résultats de cette recherche? La gratuité apparaît aux publics comme une notion équivoque, à la fois ambivalente, paradoxale et faisant difficilement sens. Elle est envisagée par eux dans une logique individuelle - ils la confrontent à leurs expériences de visite et à leurs représentations des musées et des monuments - mais également dans une logique collective - ils la considèrent alors sous la forme d'une «politique de gratuité » dont ils soulignent, unanimement, la faible adéquation aux modes réels d'approche des musées et monuments, tout en ne s'accordant pas sur sa légitimité, ses objectifs et son financement.

\section{LA GRATUITÉ : UNE NOTION ÉQUIVOQUE POUR LES PUBLICS}

Le caractère équivoque de la notion de gratuité des musées et des monuments ressort nettement dans les représentations des publics : elle est ambiguë - envisagée différemment pour soi et pour le collectif -, paradoxale et fait difficilement sens.

\section{Notion ambiguë...}

Selon qu'ils l'envisagent sur un plan collectif ou sur un plan individuel, les publics ont de la gratuité des représentations différentes : deux configurations apparaissent dans leurs discours (voir tableau 1).

Dans la première configuration, l'opposition entre une attitude favorable pour soi mais défavorable pour le collectif crée une dissonance cognitive $^{6}$ qui se concrétise par un effet d'opportunité quand la gratuité est proposée de manière exceptionnelle, catégorielle ou ponctuelle: c'est la

Tableau 1 - L'ambivalence de la gratuité repérée dans les discours : deux configurations

\begin{tabular}{|c|c|c|}
\hline & \multicolumn{2}{|c|}{ Registres de discours } \\
\hline & La gratuité pour le collectif et/ou pour les autres & La gratuité pour soi \\
\hline $\begin{array}{l}\text { Première configuration } \\
\text { (présente dans } \\
\text { l'enquête } \\
\text { quantitative, les } \\
\text { entretiens de visite } \\
\text { et les entretiens } \\
\text { individuels) }\end{array}$ & $\begin{array}{l}\text { Attitude négative } \\
\text { (émane notamment des visiteurs habitués et } \\
\text { occasionnels). } \\
\text { Revue de tous les effets négatifs (notamment risque de } \\
\text { dévalorisation des musées et monuments) et principe } \\
\text { d'équité: "les profiteurs ne sont pas les payeurs ". } \\
\text { Attitude favorable seulement si elle concerne certaines } \\
\text { catégories: familles et personnes défavorisées. }\end{array}$ & $\begin{array}{l}\text { Attitude positive } \\
\text { intention de se l'approprier, notamment si } \\
\text { la personne a une opportunité - Autant } \\
\text { que les payeurs en profitent -, si elle a } \\
\text { une expérience et si elle a de l'intérêt pour } \\
\text { la visite. } \\
\text { Les effets négatifs de la gratuité sont } \\
\text { occultés. }\end{array}$ \\
\hline $\begin{array}{l}\text { Seconde configuration } \\
\text { (présente dans les } \\
\text { entretiens individuels } \\
\text { et les entretiens de } \\
\text { groupe) }\end{array}$ & $\begin{array}{l}\text { Attitude très positive } \\
\text { (émane des habitués et occasionnels, et des non- } \\
\text { visiteurs). } \\
\text { La gratuité concerne tout le monde. Elle valorise les } \\
\text { musées et les monuments. }\end{array}$ & $\begin{array}{l}\text { Ne se sent pas concerné } \\
\text { (émane notamment des non-visiteurs). } \\
\text { Aime autant visiter les musées et les } \\
\text { monuments quand ils sont payants } \\
\text { (émane des visiteurs habitués). }\end{array}$ \\
\hline
\end{tabular}

Source : la Gratuité des musées et des monuments vue par les publics (DEPS, Ministère de la culture, 2006)

\footnotetext{
4. D'après Jodelet (1991), la représentation est une forme de connaissance socialement élaborée et partagée ayant une visée pratique et concourant à la construction d'une réalité commune à un ensemble social. Abric (1994) le définit encore comme une vision fonctionnelle du monde, qui permet à l'individu ou au groupe de donner un sens à ses conduites, et de comprendre la réalité, à travers son propre système de références, donc de s'y adapter, de s'y définir une place. Placées à la frontière du psychologique et du social, les représentations sociales permettent aux personnes et aux groupes de maîtriser leur environnement et d'agir sur celui-ci. D’après Denise JoDELET, les Représentations sociales, Paris, Puf, 1991 et Jean-Claude ABRIC, Pratiques sociales et représentations, sous la direction de J.-C. Abric, PuF, 1994,2 éd. 1997. 5. En France, on recense seulement l'étude de H. Gottesdiener et N. Godrèche à la suite des réflexions de C. Fourteau : voir Hana GotTESDIENER et Natacha GODRÈCHE, les Dimanches gratuits du musée du Louvre, enquête qualitative, Paris, Musée du Louvre-service culturel, CEREM, 1996, et Claude Fourteau, la Gratuité au bois dormant... Cinq ans de gratuité du dimanche au Louvre, 1996-2000, Paris, Musée du Louvre-service culturel, 2001. Au Royaume-Uni, l'institut MoRI a mené une intéressante étude d'impact de la gratuité sur le comportement de visite des publics, au-delà du seul indicateur de fréquentation sans aborder la question de la perception de cette gratuité. Voir Andy MARTIN, The Impact of Free Entry to Museums, MoRI, mars 2003, p. 1-6.

6. Existence de relations incompatibles entre différentes cognitions. Par « cognitions », on entend une connaissance, des opinions, une croyance sur la vie et sur soi-même ou sur son comportement. Voir A. Gombault et al., la Gratuité des musées et des monuments..., op. cit., p. 408.
} 


\section{Histoire récente de la gratuité}

\section{La résurgence de la gratuité depuis les années 1980}

Dans les années 1980, la contradiction apparente d'un musée payant se voulant pourtant démocratique a suscité un débat passionné entre partisans et adversaires de la gratuité dans le monde muséal occidental. Réglé aux États-Unis, au Canada et progressivement dans plusieurs pays d'Europe, où les musées, en fonction de leurs seuls objectifs stratégiques, choisissent ou non la gratuité pour tous et les formes qu'elle prend, le débat s'est poursuivi de façon aiguë dans les années 1990 au Royaume-Uni. En France, il a été progressivement relancé mais de façon plus modérée. En 1996, sur proposition du Ministère de la culture et de la communication, le musée du Louvre devient gratuit le premier dimanche de chaque mois, à titre expérimental pour deux ans, puis, face au succès, sans limitation de durée à partir de 1998. En 2000, la mesure est étendue à l'ensemble des musées et des monuments nationaux, suivis par plusieurs villes qui l'adoptent dans leurs musées municipaux. En 2002 enfin, les musées de la Ville de Paris offrent une gratuité permanente, bientôt rejoints par ceux de Dijon, Caen, Bordeaux ou encore du département de l'Isère. Ce retour à des mesures de gratuité en France, alors qu'elle avait progressivement disparu au $\mathrm{xx}^{\mathrm{e}}$ siècle pour laisser la place à des politiques tarifaires de plus en plus sophistiquées, a connu récemment un nouveau développement avec l'annonce d'une expérimentation de la gratuité à l'entrée dans certains musées nationaux parisiens et de province.

\section{La gratuité d'entrée des musées au Royaume-Uni}

Gratuité d'accès ou droits d'entrée, depuis plus de 20 ans, les musées du Royaume-Uni expérimentent les deux systèmes. Les leçons, parfois contradictoires, qu'ils en ont tirées ont alimenté leurs affrontements idéologiques. En 2001, Chris Smith, le ministère de la Culture, des Média et du Sport du gouvernement travailliste Blair, réussit à imposer le retour à la gratuité à l'entrée des collections permanentes dans tous les musées nationaux payants dont certains, comme le Science Museum, le Natural History Museum, le National Maritime Museum ou l'Imperial War Museum, très réticents à la mesure, étaient devenus, en réponse aux pressions financières des années 1980, des entreprises culturelles très dynamiques. La mesure conforte dans leur position les musées nationaux restés gratuits, malgré les difficultés rencontrées (notamment par le British Museum et la National Gallery). Si l'objectif de la mesure est clair - offrir le plus large accès possible aux collections nationales - et l'augmentation de la fréquentation spectaculaire, ses effets sur une réelle diversification des publics sont très vite discutés et la mise en œuvre de stratégies de prix sur l'offre périphérique dans les institutions les plus innovantes s'avère, paradoxalement, renforcée.

\section{Les arguments du débat}

Les arguments qui se sont toujours opposés dans ce débat ancien et complexe sont de plusieurs natures. Ils s'inscrivent d'abord dans un registre symbolique et politique, qui met en jeu l'identité originelle du musée (mise à la disposition de tous d'un patrimoine collectif) par opposition à son identité contemporaine (place du patrimoine dans le champ des loisirs, institutions à gérer pour qu'elles se développent, etc.). Ils relèvent ensuite d'un registre économique en se focalisant sur le coût de la gratuité. Son efficience économique est discutée, entre coût marginal nul ou réel du visiteur supplémentaire, enjeu mineur ou majeur de l'augmentation des ressources propres du musée, soutien public ou privé suffisant ou non pour maintenir un niveau d'activité approprié pour le musée. Enfin et surtout, le débat pose de façon centrale la question des publics, les partisans de la gratuité la considérant comme un idéal pour eux tandis que ses détracteurs la confrontent à des nécessités de maintien ou de développement de l'offre des musées et de sa qualité.

Les arguments connus du débat et qui concernent les publics sont trop peu convoqués dans l'élaboration et l'évaluation des politiques publiques. Principalement développés pour les musées mais valant aussi pour les monuments, ils peuvent être regroupés en cinq thèmes discutés de façon récurrente.

- Le premier concerne l'impact de la gratuité sur la fréquentation dont on sait, d'une part, qu'il est effectif à court terme mais discutable à moyen et long terme et, d'autre part, qu'il n'est pas pur mais corrélé à d'autres variables de l'offre.

- L'impact de la gratuité sur l'accessibilité du musée constitue le deuxième: celle-ci requiert la mise en place d'actions particulières pour augmenter, élargir et diversifier la fréquentation mais aussi pour accroître l'accessibilité intellectuelle et physique. Ces actions vont bien au-delà de la simple levée de barrières monétaires à l'entrée, barrières dont on sait, par ailleurs, que ce ne sont pas elles qui empêchent principalement l'accès au musée.

- Le troisième pose la question de l'équité de la gratuité : il semble que, destinée à tous, elle profite davantage aux visiteurs fidèles des musées en leur permettant des visites récurrentes, et que ces visiteurs appartiennent par ailleurs aux classes sociales les plus élevées. Si l'on est en droit de se réjouir de cette fidélisation, la question de la justice sociale n'en est pas moins posée par cette mesure qui aide les populations aisées aux dépens de celles qui sont défavorisées alors qu'elle est financée par tous. Des travaux canadiens mettent en avant que la gratuité servirait davantage la démocratie de la culture que sa démocratisation.

- L'impact de la gratuité sur les représentations que les publics ont de la valeur des musées et monuments, sur leur expérience de visite et sur leur engagement dans la visite constitue le quatrième thème: on sait que, en la matière, la gratuité a des effets contradictoires: la valeur perçue est diminuée ou augmentée selon les publics, de même que l'engagement dans la visite.

- La réalité de la gratuité est le dernier des thèmes discutés: on sait que le «prix » du musée, du point de vue du visiteur-consommateur, ce n'est pas seulement la somme à payer pour entrer, mais aussi, comme pour toute activité de loisirs et particulièrement comme pour toute activité culturelle, des coûts non monétaires : à savoir l'effort fourni pour venir et les dépenses associées à la visite et à l'effort fourni. Il a été montré par exemple que ces coûts non monétaires avaient une importance d'autant plus grande pour les publics qu'un service était gratuit, là où pourtant on penserait spontanément qu'il n'existe plus de barrières à l'entrée. L'effort fourni pour venir comporte deux dimensions : une dimension objective - le temps, la durée ou la difficulté de déplacement - et une subjective - le risque de ne pas aimer, de ne pas comprendre, d'être désapprouvé par les autres, etc. Cet élément explique en partie que la fréquentation du musée est faiblement corrélée au prix. Il a également été montré que, dans la plupart des cas, les dépenses associées à la sortie culturelle doubleraient le prix du billet. Le visiteur de musée paie un stationnement ou un titre de transport pour venir, veut aussi voir l'exposition temporaire souvent payante, boit ou mange souvent quelque chose, a peut-être envie de rapporter un produit de la boutique... tous ces éléments faisant de plus en plus partie intégrante de l'expérience de visite. La gratuité diminuerait donc théoriquement le coût monétaire global pour le visiteur de musée, sauf si la gratuité de l'entrée favorise une consommation périphérique plus importante (ce que montrent certaines études empiriques).

* Sources : Anne Gombault, "L'émergence du prix comme variable stratégique des musées: une étude internationale », dans F. Rouet (sous la dir. de), les Tarifs de la culture, op. cit., p. 165-222 et Anne GomBAULT, "Current practises in free admission to museums : an international perspective", Proceedings of the 7th International Conference on Arts management (AIMAC), Milan, Italy, Bocconi University, 2003. 
promenade qui donne l'occasion - c'était sur mon chemin, c'était gratuit, donc je suis rentré $e^{7}$, ou d'aubaine - je savais que c'était gratuit alors j'y suis allé. Pour réduire cette dissonance, certaines personnes déclarent tout à la fois qu'elles sont défavorables à la gratuité à cause de ses effets négatifs - banalisation des musées et des monuments, affluence... - et que ces effets ne sont pas démontrés, qu'elles peuvent donc, à titre personnel, profiter des situations de gratuité.

La seconde configuration montre qu'il est manifestement plus facile d'être favorable à la gratuité pour les autres que pour soi-même, posture qui appelle deux interprétations :

- soit « la gratuité s'adresserait avant tout aux plus démunis, et les visiteurs ont [eu] du mal à se reconnaître dans ceux qui disposent de peu de moyens financiers ou culturels ${ }^{8} »$; la gratuité pour les autres pourrait alors « $\mathrm{s}$ 'interpréter comme une manifestation défensive visant à préserver une certaine image de soi $^{9} \gg$. Dans ce cas, la dissonance est forte : la gratuité serait un moyen de donner accès à la culture - le fait de payer serait un obstacle -, mais c'est surtout l'intérêt qui devrait favoriser la fréquentation.

- soit la gratuité, si séduisante qu'en soit l'idée, n'est pas confortable à vivre: si, par principe, c'est « une bonne chose», le projet d'usage se heurte à des contraintes individuelles rédhibitoires. À cet égard, la position de la plupart des non-visiteurs est intéressante : plutôt favorables à la gratuité (ou à des prix bas) à titre collectif, ils ne se sentent pas concernés à titre personnel, confirmant que la mesure de gratuité qui s'adresse à eux en priorité n'est pas spontanément opérante à leur égard.

\section{... paradoxale}

La gratuité, comme le prix, recouvre pour les publics des contenus complexes et contradictoires que l'on peut résumer en quatre paradoxes :
- la gratuité des musées et des monuments existe de facto à travers les mesures tarifaires appliquées dans les lieux, mais, pour les publics, elle n'existe jamais dans l'absolu, la visite étant toujours source de coûts non monétaires. C'est donc une fausse gratuité ;

- la gratuité est une bonne chose parce que l'idée, généreuse, favorise la démocratie culturelle, mais elle est négative parce qu'elle a toujours un coût collectif en termes financiers ${ }^{10}$. À cet égard, les publics envisagent le risque de moindre entretien des lieux, de dégradation de leur conservation, et de mauvaise qualité du service pour les visiteurs comme un effet possible de la baisse des ressources propres des musées et des monuments liée à la gratuité d'entrée ;

- le fait qu'il existe des mesures de gratuité dans les musées et les monuments alors que les publics appréhendent largement ceux-ci comme des organisations culturelles insérées dans le marché, apparaît à certains comme un troisième paradoxe. Ils comprennent alors la gratuité comme une action-prix ${ }^{11}$ des musées et des monuments au service de leur stratégie de développement. Autrement dit, la vraie gratuité ne saurait exister dans un système économique de marché, puisqu'elle serait en contradiction avec lui ;

- la gratuité n'est pas équitable : seuls quelques-uns en profitent. C'est le quatrième paradoxe. Plus encore, les représentations se cristallisent sur l'idée que ceux qui en profitent le plus - les visiteurs habitués qui peuvent payer l'entrée - n'en ont pas besoin. La gratuité ne servirait donc pas la démocratisation culturelle mais l'accroissement de la consommation des amateurs. De plus, certains visiteurs jugent la mesure inique à cause du double paiement: ils financent par leurs impôts une mesure dont ils ne bénéficient pas et cette situation provoque en eux gêne ou désaccord: les profiteurs ne sont pas les payeurs. D'autres expliquent même qu'ils profitent de la gratuité pour remédier à cette iniquité.

\footnotetext{
7. Les verbatim sont présentés en italique.

8. H. GotTESDIENER et N. GodRÈche, les Dimanches gratuits du musée du Louvre..., op. cit.

9. Ibid.

10. Le fait que les représentations de la gratuité se heurtent en premier à la notion de coût direct ou indirect du service montre que le cadre de référence pour les musées et les monuments est désormais marchand. On retrouve ici ce que constatait J.-L. Sagot-Duvauroux : le coût et le financement sont les premiers réflexes d'opposition à l'établissement de la gratuité en général. En cela, les représentations de la gratuité des musées et des monuments n'auraient donc rien de spécifique. Voir Jean-Louis SAGot-Duvauroux, Pour la gratuité. Essai, Paris, Desclée de Brouwer, 1995.

11. Une action-prix consiste à fixer un niveau de prix de manière stratégique en tenant compte des contraintes que sont la réglementation, le niveau des coûts, la concurrence, et en s'appuyant sur une connaissance fine du marché et de la demande.
} 


\section{... qui fait difficilement sens}

Mais de quelle gratuité s'agit-il ? De non-paiement ou de gratuité globale? De gratuité comme offre tarifaire ou de politique de gratuité ? Les publics en effet ne savent pas clairement ce que gratuité veut dire et cette difficulté de la gratuité à faire sens est à rapprocher en partie du manque d'information sur la mesure : les personnes interrogées, quand elles en ont connaissance, la connaissent souvent vaguement. Cette difficulté peut aussi être intrinsèquement liée aux musées et monuments, qualifiés à plusieurs reprises d'offres floues (à propos desquelles) on ne sait pas à quoi s'attendre.

Dans ce contexte flou à deux titres, la gratuité est perçue comme pouvant modifier la nature même de la visite, son objectif et ses bénéfices : en n'incitant pas les visiteurs à faire l'effort d'aller jusqu'au bout de la visite, elle donnerait notamment l'idée que ce sont des lieux faciles d'accès; en leur laissant croire qu'ils ont tous les droits et en leur évitant soigneusement la question du coût, elle aurait des effets pervers sur l'intention et le comportement de visite, les visiteurs se trouvant ainsi déresponsabilisés; certaines personnes viendraient pour profiter de la gratuité sans même être intéressées par les musées et les monuments, ce qui constituerait une sorte d' " abus social ${ }^{12}$ ».

D'autres interrogations, récurrentes, portent sur les objectifs de cette gratuité et sur les effets escomptés. Quelles sont les motivations des pouvoirs publics ? À quoi sert cette mesure ? Ne traduit-elle pas un constat d'échec? Ces réactions, provenant d'une partie des personnes rencontrées, soulignent l'effet «trop beau pour être vrai » de la mesure: l'offre est suspectée d'être de qualité moindre les jours gratuits. La gratuité serait un faux problème ou peut-être une mauvaise solution, son effet sur les publics susceptibles d'en profiter serait illusoire, parce qu'elle ne ferait pas venir durablement les foules et n'accroît pas leur intérêt pour les lieux. Enfin, elle serait toujours un leurre car il faudra la payer d'une autre façon et il y aura toujours des coûts économique et culturel, pour la collectivité comme pour le visiteur.

\section{LA GRATUITÉ PENSÉE COMME PRIX}

Pour les publics, la gratuité est fortement associée au prix et au fait de payer l'entrée. La gratuité semble jouer en outre un rôle secondaire dans la décision de visite. Elle pose par ailleurs deux questions : celle de l'identité des musées et des monuments perçue par chaque individu, resituée dans un cadre de référence unique, celui de biens communs culturels dans un système marchand; et celle de la nature de la visite.

\section{Coûts monétaires et non monétaires de la gratuité}

Dans les représentations des publics, la gratuité se manifeste comme un prix composé de coûts, à la fois monétaires et non monétaires, comme un compromis entre sacrifices acceptés et bénéfices perçus. Les publics déclarent que le rôle de la gratuité, comme celui du prix, est paradoxal et relatif dans la constitution d'un projet de visite. D'abord, parce que la suppression d'un coût monétaire - le prix d'entrée - est susceptible de renforcer la perception des autres coûts monétaires engendrés par la visite : déplacement, services annexes. Ensuite, parce que la gratuité modifie également la perception que les publics ont des coûts non monétaires associés à la visite : le temps nécessaire à la visite, les efforts à fournir pour s'organiser (selon notamment que la mesure est permanente ou périodique), les coûts non monétaires que constituent l'affluence à supporter (pour certains, la foule ne constitue cependant pas un coût mais un avantage) et le risque d'être déçu par la visite, etc. Les effets de la gratuité escomptés par les publics sont étroitement associés à leur implication ${ }^{13}$ dans les musées et les monuments : ils considèrent que son impact est très dépendant de la pertinence, de l'intérêt, de l'attrait que la visite aura pour eux. L'objet de consommation n'est pas la gratuité elle-même, mais les musées et les monuments. Pour l'ensemble des publics étudiés, y compris les visiteurs habitués qui en profitent le plus, la gratuité ne fera venir personne ou si peu, l'économie qu'elle permet n'est

12. Comme il ressort de l'analyse de J.-L. Sagot-Duvauroux pour la gratuité en général, il semble que ces « abus de gratuité » sont en réalité tout à fait relatifs, mais ont un impact disproportionné dans les représentations. Voir J.-L. SAGOT-Duvauroux, Pour la gratuité. Essai, op. cit. 13. La notion d' «implication» est définie couramment comme un état non observable de motivation, d'excitation ou d'intérêt créé par un objet ou une situation spécifique. Il est possible de préciser que l'implication peut être considérée comme un trait, un état ou un processus. Il semble donc que l'implication des individus dans la visite des musées et des monuments est liée, à la fois, à un fort niveau d'intérêt qui renvoie à une composante cognitive, ainsi qu'à une importante recherche de plaisir qui suggère une composante affective. Cette distinction entre cognition et affect fait écho aux préoccupations des chercheurs qui sont partagés entre deux approches du concept d'implication : soit en termes de multiplicité de réactions cognitives, soit en termes de recherche d'expériences (par l'intérêt, l'attention et l'excitation). 
pas le problème, parce que les barrières à l'entrée sont ailleurs. Pour tous, la gratuité est déclarée secondaire dans la construction et dans la réalisation d'un projet de visite.

Pour autant, la mesure de gratuité n'est pas neutre. Elle transforme la pratique de visite de quatre façons différentes :

- elle permet d'abord de faire penser aux musées et aux monuments car elle a un effet promotionnel évident, surtout si la communication est adaptée ; - elle améliore ensuite l'accessibilité pécuniaire et symbolique pour certains segments de visiteurs, notamment les jeunes ;

- elle permet également que certains visiteurs occasionnels et habitués visitent davantage : ceux qui n'y sont pas hostiles par principe et ceux qui ne rencontrent pas d'autres barrières, comme la disponibilité en termes de temps, par exemple ;

- elle change aussi pour certains le processus de décision de visite, lequel apparaît alors plus simple, moins coûteux, à travers la notion d'essai et d'expérience. En cela, elle lève des barrières psychosociales, qui sont celles qui limitent réellement l'accès au musée.

\section{Quelles sont alors \\ les significations du paiement?}

Que la gratuité soit pensée comme un prix conduit à réfléchir sur les significations du paiement. Si la suppression des droits d'entrée autorise l'accès aux lieux pour tous, cette autorisation n'engendre pas les mêmes conséquences dans les représentations de tous les publics : pour certains, elle banalise la visite quand elle la démocratise pour d'autres. Si elle permet de s'approprier les lieux de visite, elle peut créer des effets d'aubaine ou au contraire des réactions d'évitement et modifier les repères. Si elle libère les lieux, elle peut également déresponsabiliser les individus, dégrader les conditions de visite, ou bien, au contraire, autoriser un comportement plus détendu facilitant la familiarisation avec les œuvres. Enfin, si elle modifie les représentations que les publics ont de la valeur des musées et des monuments et de leur visite, elle constitue, d'une façon générale, un risque de dévalorisation à cause des effets qui lui sont attribués, effets d'ordre symbolique (désacralisation du lieu et de la visite, diminution de la valeur perçue de l'offre) et/ou d'ordre matériel (dégradation des conditions de visite; moindre qualité perçue des services ; contraintes de planification de la visite si la gratuité est périodique). Pour certains, au contraire, en faisant des musées et des monuments des espaces partagés, non marchands, des lieux à part qui créent une convivialité et du lien social, la gratuité est un facteur de valorisation. Pour d'autres enfin, défenseurs ou détracteurs de la mesure, elle est neutre, la valeur des musées et de l'expérience de visite étant déconnectée du prix à payer.

\section{Les représentations des musées et monuments dans un contexte marchand}

D'une part, les musées et monuments sont considérés comme des objets culturels; de l'autre, la gratuité met en évidence la présence d'un rapport à l'argent: cette confrontation dessine le cadre de référence complexe dans lequel les publics insèrent musées et monuments.

Conçus comme des biens communs culturels dans un système marchand, les musées et les monuments se voient attachés des normes et des codes de visite, ce qui permet de comprendre pourquoi, dans les représentations des publics, la gratuité se manifeste comme un prix sur un marché et pourquoi le fait de payer peut être également si important pour le visiteur. Le paiement représente en effet un visa d'entrée, un engagement dans l'acte de visite et dans la valorisation de celle-ci. Les publics sont donc bien loin de partager l'idéologie originelle de la gratuité portée par de nombreux professionnels du patrimoine en France comme à l'étranger.

Dans ce cadre de référence dominant de l'échange, les réactions des publics à la gratuité sont duales : si une majorité des personnes rejette la gratuité permanente, d'autres au contraire, ayant fait une expérience positive de la gratuité, peuvent faire évoluer ce cadre de référence dans un double processus d'apprentissage de la visite et d'appropriation de la mesure de gratuité. En effet, la gratuité peut faire vivre une expérience de visite différente de celle de la configuration payante: elle modifie alors l'usage des lieux; les comportements changent et deviennent plus détendus, voire profanes, avec des variations selon le type de lieu ou de gratuité ; elle crée un contexte de convivialité spécifique et modifie le lien social inter-groupe et intragroupe. C'est une expérience collective partagée. La gratuité événementielle permet notamment des « retrouvailles communautaires » autour de l'objet patrimonial. Dans le cadre exceptionnel des visites des Journées du Patrimoine, la gratuité est par 
exemple l'occasion de vivre une expérience partagée autour d'un même objet. Ainsi, l'expérience d'une visite gratuite peut donner plus souvent lieu à de nouveaux projets de visites gratuites, voire à leur choix exclusif. L'expérience gratuite favoriserait l'appropriation individuelle des mesures de gratuité.

\section{Des représentations inscrites dans quatre univers distincts}

Si les publics partagent le fait d'associer tous les musées et les monuments à des biens communs culturels devant être accessibles à tous et faisant partie d'un patrimoine à protéger, cette vision recouvre toutefois des représentations plurielles. Celles-ci se structurent notamment à partir de l'implication et de l'attitude à l'égard des musées et des monuments, ainsi que de leur gratuité ; également à partir de la façon dont les publics perçoivent les fonctions esthétiques de ces lieux, l'utilité et les bénéfices liés à leur fréquentation ainsi que leur valeur et fonction sociales; enfin à partir de variables individuelles (les parcours de vie individuels) et à partir de l'expérience des pratiques de visite des musées et des monuments. Les représentations que les publics ont des musées et des monuments peuvent s'inscrire ainsi dans quatre univers ${ }^{14}$ différents, eux-mêmes fragmentés :

- un « univers sacré », les musées et les monuments étant perçus comme des objets extraordinaires, sacrés, universels, hors d'un environnement marchand. Cet univers se fragmente selon une vision plutôt religieuse ou plutôt laïque, selon l'attitude le plus souvent favorable à la gratuité permanente comme principe, mais parfois défavorable, et selon le niveau de pratique;

- un «univers patrimonial», les musées et les monuments étant considérés comme des biens patrimoniaux - au sens culturel mais également économique et juridique - qu'il faut respecter et transmettre. Cet univers se fragmente à partir du débat sur la gratuité et sur le mode de participation du visiteur qui doit concrétiser son engagement : la gratuité n'est pas la norme et soulève une forte tension entre accessibilité et participation financière. Apparaît ici un clivage entre les représentations des musées et des monuments - vus comme un patrimoine culturel et économique - et les représentations de leur visite - plutôt vue comme une prestation de services ;

- un « univers loisirs » - la dimension idéologique et politique des représentations est ici atténuée -, les musées et monuments étant considérés comme des lieux de loisirs qui offrent des prestations de services comme des produits culturels. C'est un univers de consommation, où la visite est mise sur le même plan que les autres loisirs. Cet univers se fragmente selon les centres d'intérêts des publics ou selon le caractère plus ou moins élitiste des lieux ou des offres ;

- un « univers étrange », où la distance qui existe entre les musées et les monuments et les publics est importante. Cette distance se caractérise par une indifférence à l'égard des lieux et/ou par une incapacité à les décrire. Les publics appartenant à cet univers sont des non-visiteurs même si tous les non-visiteurs n'y inscrivent pas leurs représentations. Cet univers se fragmente selon le caractère provisoire de la distance - certains jeunes par exemple considèrent que le patrimoine ne les concerne pas pour le moment - ainsi que sur la connaissance ou non de l'existence de codes culturels attachés à la pratique. La gratuité ne suscite pas de position affirmée et est supposée sans effet sur les pratiques.

\section{LES REPRÉSENTATIONS}

\section{DE LA POLITIQUE DE GRATUITÉ}

Les publics cherchent à comprendre les différentes mesures de gratuité dans le cadre d'une politique culturelle, d'une politique tarifaire ou de gratuité qu'ils n'identifient pas. Les représentations qu'ils en ont convergent fortement autour d'une idée principale: quel que soit le registre mobilisé cette politique serait peu adaptée, ce qui renforcerait leur sentiment que l'offre, souvent déjà mal identifiée, est floue. Leurs critiques se focalisent sur deux points : manque de cohérence et manque de communication. Par ailleurs, les représentations des publics divergent, et ce fortement, lorsqu'il s'agit de définir ce que devraient être les objectifs et le financement de cette politique. 


\section{Manque de cohérence...}

La politique de gratuité apparaît souvent aux publics comme incohérente : attirant trop de monde en même temps, la gratuité crée des problèmes de gestion des flux et le service perd de sa qualité en raison d'un surcroît de travail du personnel; elle ne serait pas pour autant capable d'augmenter la fréquentation de manière durable et significative, ce qui pose la question de son efficacité et de sa légitimité. La politique actuelle ne serait ainsi qu'un premier pas, une preuve de bonne intention, un beau geste.

La pertinence de cette politique de gratuité est en outre mise en cause. De façon générale, les publics lui reprochent de mal prendre en compte leur comportement de visite : ils suggèrent d'élargir les plages de gratuité pour en rendre le bénéfice plus aisé ; de veiller à la gestion des flux et à la qualité du service; de proposer des prix adaptés à chaque segment de publics, avec des mesures de gratuité catégorielles (pour les jeunes, les scolaires, les personnes âgées à faibles ressources économiques), voire des mesures périodiques pour les résidents locaux ou nationaux.

Dans le contexte de ces représentations, nombreuses sont les questions portant sur le coût des mesures de gratuité. Toutefois certains souhaits exprimés - que les enfants par exemple bénéficient de tarifs bas - traduisent la méconnaissance que les publics ont des mesures de gratuité puisque la gratuité est permanente pour les moins de 18 ans dans les musées et monuments nationaux. Cette méconnaissance serait bien, de l'avis de certains, la conséquence d'une mauvaise communication.

\section{... de communication}

C'est de façon unanime que les publics soulignent les déficiences en matière de communication, déficiences à la fois quantitatives et qualitatives. La publicité des mesures de gratuité périodique est jugée insuffisante, confuse et mal ciblée. Si la plupart des publics connaissent par exemple l'existence de la gratuité des visites à l'occasion des Journées du Patrimoine et l'évoquent spontanément, beaucoup ignorent les autres mesures de même nature; certains en ont vaguement entendu parler et un petit nombre seulement en est informé. Certains regrettent de n'avoir pas eu connaissance par voie de presse des dimanches gratuits ni d'avoir été informés de l'arrêt au niveau local d'une mesure de gratuité. Déjà en 2001, ce constat avait été fait à l'occasion d'une enquête sur la notoriété de la gratuité, selon laquelle $35 \%$ seulement des Français déclaraient être au courant de la gratuité dans les musées et $25 \%$ dans les monuments ${ }^{15}$.

\section{Une légitimité mise en cause}

Si les publics s'accordent sur l'inadaptation de la politique de gratuité, ils se divisent quant à sa légitimité, ses objectifs et ses modalités (voir tableau 2, p. 9).

La légitimité de la gratuité est défendue par une partie des publics habitués, qui inscrivent leurs représentations dans l'univers sacré des musées et des monuments : pour ces «fondamentalistes », la gratuité est inhérente à l'identité originelle même des musées et des monuments et devrait être permanente. Une partie des publics - les «opposants »-, en déclarant ouvertement que les musées et les monuments n'ont pas à être gratuits, remettent en question cette légitimité de la gratuité. À l'appui de ce rejet, ces personnes invoquent le risque d'assistanat des services publics, risque qui ferait oublier la notion d'effort tant sur le plan financier que culturel. Est-ce qu'on ne va pas encore vers une politique sociale, qui serait intéressante mais pas forcément valorisante pour l'individu quel qu'il soit? [...] Cela ne peut valoriser l'homme quel qu'il soit [...] de rendre les choses gratuites, déclare un visiteur occasionnel. Pour principal argument à l'encontre d'une mesure de gratuité permanente, les opposants avancent que les musées et les monuments doivent être sauvegardés, conservés et entretenus. La réticence à la gratuité ne signifie donc pas qu'il y a réticence à l'égard de l'accessibilité mais souligne plutôt la difficulté de concilier gratuité permanente et protection du patrimoine. Ceci explique également la position des publics « mitigés », qui, plutôt qu'une gratuité permanente, souhaitent une gratuité ciblée, c'est-à-dire des mesures ponctuelles et/ou catégorielles. Ils

15. Notons à ce propos que le fameux bouche-à-oreille, reconnu comme un vecteur majeur de la communication culturelle, notamment pour le cinéma et la musique, ne semble pas fonctionner dans le cas des musées et des monuments, peut-être parce que l'expérience de consommation y est moins populaire. Voir S. OCTOBRE et F. RouET, «La gratuité du premier dimanche du mois dans les monuments et musées nationaux », chap. cité. 
Tableau 2 - Représentations de la légitimité de la gratuité dans les musées et les monuments et représentations liées de la politique de gratuité

\begin{tabular}{|c|c|c|}
\hline & $\begin{array}{c}\text { Représentations } \\
\text { de la légitimité de la } \\
\text { gratuité dans les musées } \\
\text { et les monuments }\end{array}$ & $\begin{array}{l}\text { Représentations liées à la politique de gratuité } \\
\text { des musées et des monuments }\end{array}$ \\
\hline $\begin{array}{l}\text { Les } \\
\text { fondamentalistes } \\
\text { univers sacré }\end{array}$ & $\begin{array}{l}\text { La gratuité est inhérente } \\
\text { aux musées et aux } \\
\text { monuments }\end{array}$ & $\begin{array}{l}\text { Une obligation, un devoir pour un patrimoine collectif. } \\
\text { Un service public. Un outil de démocratisation culturelle. La gratuité devrait être } \\
\text { permanente. } \\
\text { Une obligation, un devoir. C'est un patrimoine qui appartient à tous. On paie des } \\
\text { impôts. Des gens ont fait des donations. [...] II faut ouvrir la connaissance à tout le } \\
\text { monde. } \\
\text { - J'associe gratuité à patrimoine. } \\
\text { Extraits des entretiens individuels } \\
\text { (visiteurs occasionnels) } \\
\text { - Moi je suis assez d'accord sur la notion de gratuité pour ce qu'on appelle les } \\
\text { musées nationaux ou le patrimoine parce que, comme le nom l'indique, ça } \\
\text { appartient à l'État donc aux Français. [...] } \\
\text { - J'associe le mot démocratisation à la gratuité. } \\
\text { (visiteurs habitués) }\end{array}$ \\
\hline $\begin{array}{l}\text { Les mitigés } \\
\text { univers } \\
\text { patrimonial }\end{array}$ & $\begin{array}{l}\text { La gratuité des musées et } \\
\text { des monuments est une } \\
\text { bonne chose mais sa mise } \\
\text { en œuvre est délicate }\end{array}$ & $\begin{array}{l}\text { Elle permet de réduire les inégalités sociales, mais elle est coûteuse. Il faut aussi } \\
\text { entretenir le patrimoine. La gratuité doit rester occasionnelle. } \\
\text { La gratuité, c'est bien gentil, mais la première idée qui me vient est ce que cela } \\
\text { coûte; c'est toujours la même chose, il faut bien entretenir... et s'il y a pas d'entrées } \\
\text { payantes... } \\
\text { Extraits des entretiens individuels } \\
\text { Elle est efficace si elle est ciblée : elle est appréciée pour les jeunes (enfants, } \\
\text { scolaires, étudiants) et les familles. } \\
\text { - [l'accès pour] Les personnes qui n'auraient pas forcément les moyens d'y aller } \\
\text { [dans les musées et les monuments] } \\
\text { - Oui, les familles complètes. Pour les familles nombreuses, c'est onéreux [...] } \\
\text { - Je pense que la gratuité, c'est plus pour les familles peut-être. Les familles où il y a } \\
\text { eu beaucoup d'enfants. } \\
\text { (visiteurs occasionnels) } \\
\text { Elle est efficace si elle vient en complément d'une politique d'éducation culturelle. } \\
\text { - L'accompagnement, c'est absolument nécessaire [...] } \\
\text { - Que ce soit gratuit, amener quelqu'un devant un tableau, si il n'y a personne pour } \\
\text { sensibiliser... } \\
\text { (visiteurs habitués) } \\
\text { Extraits des entretiens de groupe }\end{array}$ \\
\hline $\begin{array}{l}\text { Les opposants } \\
\text { univers loisirs }\end{array}$ & $\begin{array}{l}\text { Les musées et les } \\
\text { monuments n'ont pas à } \\
\text { être gratuits }\end{array}$ & $\begin{array}{l}\text { Elle est coûteuse, inefficace, laxiste. } \\
\text { - Bon je vous dis, il y a de l'abus social, on peut avoir des gens dans toute cette } \\
\text { foule de badauds qui va y aller sans but et sans idée, il peut y en avoir quelques- } \\
\text { uns qui accrochent, hein. [...] Mais à mon avis ça restera une minorité. Les gens, } \\
\text { je suis persuadé que si vous faites une enquête huit jours après y'en aura neuf sur } \\
\text { dix des gens qui n'y seront pas allés volontairement, qui y seront allés uniquement } \\
\text { parce que c'est gratuit. } 89 \text { ou } 90 \% \text { n'auront même plus idée de ce qu'ils ont vu. } \\
\text { [...] Ils sont, ils seront passés dans les allées sans, sans les retenir. Donc à ce } \\
\text { moment-là, c'est un peu gâcher une gratuité pour ces gens-là. } \\
\text { Extraits des entretiens individuels }\end{array}$ \\
\hline
\end{tabular}

Source : la Gratuité des musées et des monuments côté publics (DEPS, Ministère de la culture, 2006)

invoquent alors l'effet « déclic » de ce type de gratuité, laquelle, en créant l'occasion ou la surprise, permettrait, plus qu'une gratuité permanente qui deviendrait routinière, d'initier, d'éduquer, d'attirer les publics. De la même façon, pour fidéliser les visiteurs, ils déclarent préférer les cartes forfaitaires (passeport, forfait...) à la gratuité permanente.

\section{Des objectifs mal compris}

Les publics sont divisés également quant à la façon de se représenter les objectifs de la politique de gratuité dans les musées et les monuments : ils perçoivent l'instauration de mesures de gratuité comme étant motivée par la volonté d'augmenter la 
fréquentation, de faire venir plus de monde, en levant un frein financier. Ils émettent des jugements de valeur affirmés sur cette volonté, soit positifs - ouvrir les musées et les monuments à tous; réduire les inégalités sociales; faire connaître l'histoire -, soit négatifs - obliger les personnes à revenir pour voir ; gratuité démagogique ou gratuité qui permet de faire du chiffre d'affaires avec les produits dérivés ou encore un effet d'annonce.

\section{Des modes de financement discutés}

Une même différenciation des représentations se retrouve quant aux modes de financement de la gratuité : certains prônent les financements privés (mécénat, parrainage, fondations, tourisme, produits dérivés) alors que d'autres les dénoncent, craignant qu'ils ne soient dangereux pour l'indépendance des choix culturels et leur préférant un financement public classique par l'impôt, soit par conviction - à mesure collective, financement collectif -, soit par réalisme vu l'insuffisance des autres financements. Ils sont fortement contestés par les partisans du financement privé au motif que la collectivité n'a pas à supporter une mesure profitant seulement à quelques-uns.

Dans les deux cas, la même question : qui va payer pour financer la politique de gratuité ? L'État? Les visiteurs? Les touristes ? Les mécènes et parrains ?... Selon certains, la gratuité peut être pensée dans une stratégie marketing : elle générerait un volume d'activités périphériques plus élevé que si les visiteurs acquittaient des droits d'entrée ; les revenus de ces activités contribueraient à la financer, au moins en partie. Effet dont la réalité elle-même est discutée, comme est discutée la légitimité de ce qui apparaît à certains comme une marchandisation de la culture.

\section{DEs PISTES POUR L'ACTION}

La gratuité pose systématiquement la question de son principe autant que celle de son projet d'usage et de son usage réel, par son caractère équivoque et vu le rôle secondaire que les publics lui attribuent dans leur décision de visite et sa réalisation.

Dans une perspective collective, l'analyse des représentations des publics révèle que la gratuité constitue autant une mesure à valeur symbolique, de nature politique, qu'une mesure tarifaire pragmatique, une «action-prix » au service des objectifs stratégiques des musées et des monuments. Cela se traduit par une polarisation forte des attitudes: pour/contre, favorable/défavorable. Dans une perspective individuelle (la gratuité pour soi), la prise de position est moins tranchée. L'examen de la relation entre attitude vis-à-vis de la gratuité, projet d'usage et usage fait apparaître d'autres éléments, parmi lesquels les formes de la gratuité, les circonstances dans lesquelles elle est proposée, les pratiques des musées et des monuments, etc. La cohérence entre ces deux attitudes - la gratuité pour le collectif ou la gratuité pour soi - est faible.

Dans leurs représentations, les publics reconnaissent les musées et les monuments comme une valeur collective symbolique d'un patrimoine ou d'une culture, mais ils les perçoivent d'abord comme des biens culturels communs, intégrés dans un environnement marchand. L'importance traditionnelle accordée par les professionnels du secteur à la seule «valeur d'existence ${ }^{16}$ » de ces institutions est relative pour les publics qui leur attribuent davantage une valeur d'usage et d'échange. Pour les musées et les monuments, c'est un élément important de changement d'identité que la communication auprès des publics gagnerait à prendre en compte.

\section{L'implication, variable centrale de la réflexion}

Bien qu'elle ne figure pas au premier plan dans les débats sur la gratuité, l'implication dans la visite apparaît nettement comme une variable « cachée » importante en matière de représentations des musées et des monuments. Ceux-ci peuvent agir sur cette variable afin de favoriser leur accessibilité, notamment à travers des mesures de gratuité, pourvu que ces mesures soient intégrées de façon cohérente à la stratégie de chaque site (ou groupe de sites) et non décidées de manière exogène sans prise en compte de la segmentation des publics. Dans ce contexte, les pistes d'action sont à rechercher dans les trois dimensions constitutives de l'implication dans la visite : la pertinence, l'intérêt et l'attirance. 


\section{La pertinence de la visite...}

Si les publics estiment que les visites sont pertinentes, utiles et importantes, ils l'expriment de façon floue et peu argumentée. Cette activité apparaît comme quelque chose dont on n'a pas besoin dans la vie quotidienne. Cette dimension de l'implication demeure centrale, notamment à l'égard des non-visiteurs et des visiteurs occasionnels, pour lesquels demeurent sans réponse des questions comme : à quoi sert la culture ? Pourquoi visiter un musée ou un monument? En quoi est-ce utile ? Or, des réponses à ces questions peuvent se décliner plus aisément dans le cadre d'un site ou d'un musée que de façon globale.

\section{... son intérêt}

Pour que se dessine l'intérêt pour l'objet patrimonial, il faut qu'une expérimentation de l'activité de visite ait lieu; la fonction «première fois » est quasiment uniquement réalisée dans le cadre des visites scolaires pour les publics les moins avantagés. Les représentations négatives qu'en ont les non-visiteurs indiquent que cette action de primosocialisation à la culture par le système scolaire n'est pas nécessairement la plus pertinente et/ou semble mal conduite. À rebours, favoriser la visite avec le cercle familial ou amical proche, par exemple par des billets d'entrée gratuite annuelle pour la famille, permet de combiner les bénéfices de la gratuité - individuelle et personnalisée - et ceux d'une première expérience au sein d'un cercle de sociabilité élective. Plus largement, toutes les actions qui permettent d'augmenter la fréquence des visites en famille ou entre amis et d'améliorer ainsi la socialisation culturelle des publics de demain feront croître leur niveau d'intérêt.

\section{... et son attractivité}

L'attirance des publics pour les musées et les monuments suppose la mise en œuvre des techniques qui permettent de faire de la visite une expérience. Il s'agit que ces lieux soient plus animés et plus vivants en acceptant qu'ils s'inscrivent aussi dans le champ des loisirs. Ce positionnement est nécessaire pour de nombreux visiteurs souhaitant être stimulés sur le plan sensoriel, recherchant de l'animation et tout ce qui favoriserait leur rencontre avec les collections et les lieux. C'est aussi ce sens que prend le marketing événementiel des musées et des monuments les plus dynamiques qui se développe dans de nombreux pays.

\section{Les formes de la gratuité : quelques exemples}

Les offres de gratuité, de même que l'ensemble des politiques tarifaires des musées et des monuments sont de plus en plus pensées dans le cadre de ce travail sur l'implication des publics.

- Les Journées du Patrimoine plébiscitées par les publics sont perçues comme un événement en lui-même qui attire, la gratuité constituant seulement une modalité de production de cet événement. La promotion de ces Journées est de loin plus importante que leur gratuité : la forte publicité, à travers notamment la presse audiovisuelle, touche le grand public, ce qui l'amène à regretter le déficit de communication autour des autres mesures de gratuité et plus généralement autour des musées et des monuments.

-D'autres mesures de gratuité catégorielles existent : par exemple celles qui concernent les enfants et les jeunes en général avant leur entrée dans la vie active, la gratuité constituant en effet pour eux un élément attirant de l'offre - même si elle est insuffisante puisqu'ils disposent de temps mais manquent de ressources économiques.

\section{La segmentation des publics}

Pour accroître l'implication des publics, il convient prioritairement de tenir compte de leur diversité. La segmentation n'est plus seulement à faire par niveau de pratique, de catégorie socioprofessionnelle, ou par origine géographique, mais s'applique aux variables individuelles qui jouent désormais pleinement dans le comportement des publics, comme par exemple le degré d'individualisme et la relation à l'autre (quête d'individualisation et de personnalisation de la relation aux musées et aux monuments, ou bien recherche de fusion sociale, animée d'un fort désir d'appartenance « à un groupe d'un instant»). À chaque segment, un prix est possible qui positionne l'offre.

\section{Une politique globale ou différenciée ?}

Une politique de gratuité pour les musées et les monuments peut-elle être pensée de façon globale alors que tout différencie les lieux - identité et missions, histoire, taille, situation géographique, fréquentation, stratégies -, et alors que ces lieux évoluent désormais dans un contexte de concurrence entre activités de loisirs? Mettre en place des mesures identiques dans des lieux qui ne le sont pas entretient l'impression de manque de cohérence, et, peut-être paradoxalement, de complexité. Variable plus endogène qu'exogène, le prix des musées et des monuments relève aujourd'hui d'une logique de marché et non pas de la seule logique politique : il doit donc pouvoir constituer un réel instrument de gestion stratégique du musée ou du monument au service de ses objectifs culturels, marketing et financiers propres. 
C'est dans le cadre de la mise en œuvre de stratégies de prix autonomes, différentes selon l'identité des musées et des monuments, et donc selon la stratégie des établissements, que les mesures de gratuité - permanente, catégorielle, événementielle peuvent sans doute atteindre une réelle efficacité. Qu'elles favorisent la promotion de l'offre ou permettent l'accès pécuniaire et/ou symbolique de cer- tains publics à cette offre, ces mesures n'ont de sens que si elles sont définies en fonction des caractéristiques des publics, de leurs attentes et comportements de visite. Des stratégies de prix autonomes n'excluent cependant pas des actions communes suivant le principe de la coopétition ${ }^{17}$ permettant de mieux faire face à la concurrence d'autres activités de loisirs.

17. Néologisme né de la combinaison des mots «coopération» et «compétition », la coopétition désigne le fait que, dans un environnement complexe, les organisations peuvent être amenées à coopérer sur certains points tout en se concurrençant sur d'autres.

\section{RÉSUMÉ}

La question de la gratuité a engendré de nombreux débats mettant en scène positions des professionnels de la culture, effets sur les volumes mesurés de fréquentation et sur la gestion des musées et des monuments. Une question a été laissée dans l'ombre : celle de la réception des mesures de gratuité par les publics destinataires. L'analyse des représentations de ces mesures, que l'on découvre multiples, contradictoires et peu congruentes avec les idéaux portés par les institutions elles-mêmes, met en évidence toute la subtilité du jeu d'interactions entre mesure tarifaire, compréhension d'une politique culturelle, impact sur les représentations des musées et des monuments et fréquentation de ces lieux. Elle trace de ce fait des pistes de renouvellement du marketing de l'offre des mesures de gratuité, tant en termes de valorisation des contenus culturels proposés que de positionnement, de segmentation et de communication de ces mesures.

\section{ABSTRACT}

The question of free admission has generated numerous debates, highlighting various viewpoints of professionals involved in Culture, effects on the measured volumes of attendance and on the management of museums and monuments. One question has been left aside: that of the reception of free admission measures by the destined audiences. The analysis of the representations of these measures, which are found to be multiple, contradictory and not very congruent with the ideals put forth by the institutions themselves, shows the subtlety of the complex interactions between the tariff amount, the comprehension of a cultural policy, impact on the representations of museums and monuments and frequentation of these venues. This analysis thus points to a renewal of the marketing of free admission offers, in terms of the validation of proposed cultural content as well as through positioning, segmentation and communication of these measures. 